YITP-12-36

\title{
Curvature Perturbation Spectrum in Two-field Inflation with a Turning Trajectory
}

\author{
Shi Pi* \\ Department of Physics and \\ State Key Laboratory of Nuclear Physics and Technology, \\ Peking University, Beijing 100871, China \\ Misao Sasakit \\ Yukawa Institute for Theoretical Physics, \\ Kyoto University, Kyoto 606-8502, Japan
}

\begin{abstract}
We revisit a two-component inflaton model with a turning trajectory in the field space, where the field slowly rolls down along the trajectory. We consider the case when the effective mass in the direction perpendicular to the trajectory, namely the isocurvature direction, is either of the same order as or much larger than the Hubble parameter. Assuming that the turning angular velocity is small, we compute analytically the corrections to the power spectrum of curvature perturbation caused by the mediation of the heavy isocurvature perturbation, and compare our analytic results with the numerical ones. Especially, when $M_{\text {eff }}^{2} \gg H^{2}$, we find that it is proportional to $M_{\text {eff }}^{-2}$. This result is consistent with the one obtained previously by an effective field theory approach.
\end{abstract}

PACS numbers: $98.80 . \mathrm{Cq}, 04.50 . \mathrm{Kd}$

*Electronic address: spi@pku.edu.cn

${ }^{\dagger}$ Electronic address: misao@yukawa.kyoto-u.ac.jp 


\section{INTRODUCTION}

Inflation [1, 2] has been recognized as the most competitive model for the early universe, which can not only solve basic problems of big-bang cosmology, say, the horizon and flatness problems and overproduction of topological defects, but also give a natural explanation for the primordial fluctuations that account for the observed CMB anisotropy and large scale structure of the universe.

The simplest model of inflation is driven by a single scalar field with a canonical kinetic term and sufficiently flat potential, which generates an almost scale-invariant, highly Gaussian curvature perturbation 20]. But one can still consider a single-field model with complex kinetic term like k-inflation [18] or DBI inflation [10]. The WMAP has accurately confirmed the existence of primordial curvature perturbations with a nearly scale-invariant spectrum of order $10^{-9}$, but at the same time indicated the possible existence of non-Gaussianity that cannot be explained by a single-field slow-roll inflation with canonical kinetic term [3].

Multi-field inflation is another natural generalization of the simplest single-field inflation. It is motivated partly because of some theoretical considerations, like string landscape [4], and partly because it can lead to more abundant phenomena. For example, N-flation or assisted inflation [9] shows that $N$ scalar fields move collectively to sustain inflation to an e-folding number required, even if each individual field is unable to drive inflation. Also one can consider multi-field k-inflation or DBI inflation [11 13$]$.

As a case study, two-field inflation can bring us fruitful new properties while maintaining geometrical intuitions in the field space. In slow-roll paradigm, we can classify two-field models into different categories by their masses, velocity in field space, etc.. The simplest case is when both fields are almost massless. In this case the effect of the curvature of the trajectory in field space can be neglected, implying that the equations of motion can be effectively decomposed into the one along the trajectory and the other orthogonal to the trajectory. One then call the field along the trajectory the "adiabatic" component and the one orthogonal to it the "entropy" or "isocurvature" component. The curvature perturbation (literally speaking the curvature perturbation on comoving or uniform density slices) is directly related to the adiabatic component of the field perturbation (on flat slices), and is affected by the entropy perturbation via "transfer function", which can be calculated in super-horizon era 14 -17]. Or, one can calculate directly the power spectrum by using the $\delta N$ formalism [23 26].

Another important case is when the trajectory is along a deep valley, that is, the effective mass along the trajectory is sufficiently small so that the field is in slow-roll motion, while the effective isocurvature mass perpendicular to the trajectory is heavy, $M_{\text {eff }} \gg H$. In such a model, the behavior is more or less like that in a single-field theory since the isocurvature perturbation quickly decays out. Namely, integrating out the heavy field, the system can be described by an effective single field theory. In this effective field approach, it has been recently claimed that the effect of the heavy field can be absorbed into a correction in the sound speed which is inversely proportional to the squared effective mass of the isocurvature component [36 38]. However, because of some non-trivial assumptions in this approach it is desirable to compute the correction without resorting to the effective field approach, and justify or falsify it.

The case that adjoins these models is called "quasi-single field" inflation by [34], in which the effective mass of isocurvature mode $M_{\text {eff }}$ is of the same order as the Hubble parameter

$H$. Assuming for simplicity that the field is approximately in a circular motion with a 
small and constant angular velocity, one can use the in-in formulism [5] 7] to calculate the 2-point function of the curvature perturbation by quantizing the curvature and isocurvature perturbations as free fields, with the coupling terms as interaction vertices [19, 35]. They calculated the correction to power spectrum in $M_{\text {eff }} \sim H$ case, and found it proportional to the angular velocity squared with a $M_{\text {eff-dependent coefficient of order } 1 \text {, which was }}$ estimated by numerical methods [35].

The aim of the current paper is to apply the method developed in the "constant turn" quasi-single field inflation in [34] to calculate analytically the coefficient of the corrections caused by the mediation of isocurvaton to the power spectrum. Especially for the case when the effective mass of the isocurvature mode is large, $M_{\mathrm{eff}}^{2} \gg H^{2}$, we examine the result obtained by the effective field theory approach [36, 38], which is found to be consistent with our analytic result.

This paper is organized as follows. In Section [I] we briefly review the quasi-single field inflation and the in-in formulism used to calculate the power spectrum of curvature perturbation, and calculate its coefficient analytically. In Section III we extend our calculation to the case of a heavy isocurvature mode, and compare our result with the one obtained by a totally different method, namely, the effective field approach in which the heavy field is integrated out from the beginning. We conclude our paper in Section IV. Some detailed calculations are spelled out in Appendix.

\section{LIGHT ISOCURVATURE MODES: QUASI-SINGLE FIELD INFLATION}

In this section we give a brief review of a simple two-field inflation model: the quasi-single field inflation. Using the in-in formalism, we derive corrections to the power spectrum of the curvature perturbation due to interactions with the isocurvature mode.

Quasi-single field inflation describes a segment of time during generic inflation when the curvature field is massless and undergoes a slow-roll trajectory, while the isocurvature mode has mass of order $H$. To be specific, we consider a motion along an arc with radius $R$. Then we can naturally decompose the field into the curvature mode along the tangent of the circle, $R \theta$, and the isocurvature mode given by the radial field $\sigma$. We call $\sigma$ the isocurvaton. The Lagrangian of such a system is

$$
S_{m}=\int d^{4} x \sqrt{-g}\left[-\frac{1}{2}(\tilde{R}+\sigma)^{2} g^{\mu \nu} \partial_{\mu} \theta \partial_{\nu} \theta-\frac{1}{2} g^{\mu \nu} \partial_{\mu} \sigma \partial_{\nu} \sigma-V_{\mathrm{sr}}(\theta)-V(\sigma)\right]
$$

where $V_{\text {sr }}(\theta)$ is a usual slow-roll potential, $V(\sigma)$ is a potential that forms a circular valley and traps the isocurvaton at $\sigma=\sigma_{0}$.

The equations of motion for classical trajectory is then

$$
\begin{aligned}
3 M_{p}^{2} H^{2} & =\frac{1}{2} R^{2} \dot{\theta}_{0}^{2}+V+V_{\mathrm{sr}}, \\
-2 M_{p}^{2} \dot{H} & =R^{2} \dot{\theta}_{0}^{2}, \\
0 & =R^{2} \ddot{\theta}_{0}+3 R^{2} H \dot{\theta}_{0}+V_{\mathrm{sr}}^{\prime}, \\
0 & =\ddot{\sigma}_{0}+3 H \dot{\sigma}_{0}+V^{\prime}-R^{2} \dot{\theta}_{0}^{2},
\end{aligned}
$$

where $R \equiv \tilde{R}+\sigma_{0}$. Now $\dot{\sigma}_{0}=0$ gives us $V^{\prime}\left(\sigma_{0}\right)=R \dot{\theta}_{0}^{2}$. This relation shows that the field is not exactly at the bottom of the valley of the potential $V$, but is slightly shifted from it to 
provide the centripetal force of circular motion. In what follows we omit the argument $\sigma_{0}$ of $V^{\prime}, V^{\prime \prime}, V^{\prime \prime \prime}$, etc., unless there is a chance of confusion. We denote the "rotation speed" by $\dot{\theta}$ as in [15] with a minus sign because of the different orientation. In the effective-field-theory papers [38, 40, 41], they used $\eta_{\perp}=\dot{\theta} / H$ instead. We define the slow-roll parameters along the trajectory as

$$
\begin{aligned}
& \epsilon \equiv-\frac{\dot{H}}{H^{2}}=\frac{R^{2} \dot{\theta}_{0}^{2}}{2 H^{2} M_{\mathrm{Pl}}^{2}} \approx \frac{M_{\mathrm{Pl}}^{2}}{2}\left(\frac{V_{\mathrm{sr}}^{\prime}}{R V_{\mathrm{sr}}}\right)^{2}, \\
& \eta \equiv \frac{\dot{\epsilon}}{H \epsilon} \approx-2 M_{\mathrm{Pl}}^{2} \frac{V_{\mathrm{sr}}^{\prime \prime}}{R^{2} V_{\mathrm{sr}}}+2 M_{\mathrm{Pl}}^{2}\left(\frac{V_{\mathrm{sr}}^{\prime}}{R V_{\mathrm{sr}}}\right)^{2} .
\end{aligned}
$$

We will not define the "slow-roll parameters" perpendicular to the trajectory since the field does not slowly roll in this direction, and since they can be represent by $\dot{\theta}_{0}$ and $V^{\prime \prime}$.

Now let us consider the perturbation to $\theta$ and $\sigma$ in the spatially flat gauge where

$$
h_{i j}=a^{2}(t) \delta_{i j}
$$

and

$$
\theta(t, \mathbf{x})=\theta_{0}(t)+\delta \theta(t, \mathbf{x}), \quad \sigma(t, \mathbf{x})=\sigma_{0}+\delta \sigma(t, \mathbf{x}) .
$$

In this gauge in the leading order in the slow-roll approximation, the gravitational effect can be neglected, and we obtain the Hamiltonian density,

$$
\begin{aligned}
\mathcal{H}_{0} & =a^{3}\left[\frac{1}{2} R^{2} \dot{\delta} \dot{\theta}_{I}^{2}+\frac{R^{2}}{2 a^{2}}\left(\partial_{i} \delta \theta\right)^{2}+\frac{1}{2} \dot{\delta \sigma^{2}}+\frac{1}{2 a^{2}}\left(\partial_{i} \delta \sigma\right)^{2}+\frac{1}{2} M_{\mathrm{eff}}^{2} \delta \sigma^{2}\right], \\
\mathcal{H}_{2}^{I} & =-c_{2} a^{3} \delta \sigma \dot{\delta} \theta, \quad c_{2}=2 R \dot{\theta}, \\
\mathcal{H}_{3}^{I} & =-a^{3} R \delta \sigma \dot{\delta} \dot{\theta}^{2}-a^{3} \dot{\theta} \dot{\delta} \theta \delta \sigma^{2}+a R \delta \sigma\left(\partial_{i} \delta \theta\right)^{2}+\frac{a^{3}}{6} V^{\prime \prime \prime} \delta \sigma^{3}, \\
M_{\mathrm{eff}}^{2} & =V^{\prime \prime}+3 \dot{\theta}^{2}
\end{aligned}
$$

where and below we omit the subscript 0 from the background quantities. It is known that the conserved curvature perturbation on comoving slices $\mathcal{R}_{c}$ is given in terms of the field fluctuation in the flat slices along the trajectory $\delta \theta$ as [21, 22]

$$
\mathcal{R}_{c}=-\frac{H \delta \theta}{\dot{\theta}}
$$

For the "constant turn" case, $c_{2}$ and $M_{\text {eff }}^{2}$ are both constants. $M_{\text {eff }}$ is the effective mass of the isocurvature field. We split the Hamiltonian into the free part $\mathcal{H}_{0}$ and interacting parts $\mathcal{H}_{2}, \mathcal{H}_{3}$. Next we will treat $\mathcal{H}_{2}$ as interacting vertex to the free Hamiltonian $\mathcal{H}_{0}$ to calculate the power spectrum, while this vertex is proportional to $\dot{\theta}_{0} / H$ and depicted in Fig. 1, $\mathcal{H}_{3}$ is only useful when turning to investigate the bispectrum which is not investigated in the present paper. This method is valid only when the interacting Hamiltonian is small, i.e.,

$$
\left(\frac{\dot{\theta}}{H}\right)^{2} \ll 1, \quad \frac{\left|V^{\prime \prime \prime}\right|}{H} \ll 1 .
$$




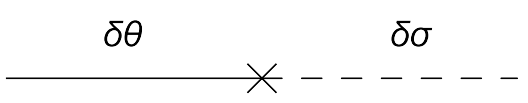

FIG. 1: The second order interacting vertex $\mathcal{H}_{2}$ which is proportional to $\dot{\theta} / H$.

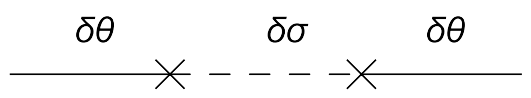

FIG. 2: The leading order to the 2-point function of curvature perturbation, mediated by a isocurvature field.

Then the perturbation theory can be used, and there is no constraint on the value of $M_{\text {eff }} \sim$ $V^{\prime \prime}$ : It maybe small, of order $H$, or large. The terminology "quasi-single field" denotes the case $M_{\text {eff }} \sim H$ which we review in this section. The case when $M_{\text {eff }} \gg H$ will be studied in Section III by the same approach.

In the interaction picture, we quantize the Fourier components $\delta \theta_{\mathbf{k}}^{I}$ and $\delta \sigma_{\mathbf{k}}^{I}$ of the free fields $\delta \theta^{I}$ and $\delta \sigma^{I}$,

$$
\begin{aligned}
\delta \theta_{\mathbf{k}}^{I} & =u_{\mathbf{k}} a_{\mathbf{k}}+u_{-\mathbf{k}}^{*} a_{-\mathbf{k}}^{\dagger}, \\
\delta \sigma_{\mathbf{k}}^{I} & =v_{\mathbf{k}} b_{\mathbf{k}}+v_{-\mathbf{k}}^{*} b_{-\mathbf{k}}^{\dagger},
\end{aligned}
$$

where $a_{\mathbf{k}}$ and $b_{\mathbf{k}}$ are the annihilation operators of $\delta \sigma$ and $\delta \theta$, respectively, and each one satisfies the canonical commutation relation,

$$
\left[a_{\mathbf{k}}, a_{-\mathbf{k}^{\prime}}^{\dagger}\right]=(2 \pi)^{3} \delta^{3}\left(\mathbf{k}+\mathbf{k}^{\prime}\right), \quad\left[b_{\mathbf{k}}, b_{-\mathbf{k}^{\prime}}^{\dagger}\right]=(2 \pi)^{3} \delta^{3}\left(\mathbf{k}+\mathbf{k}^{\prime}\right)
$$

with all the other commutators equal to zero. The mode functions, $u_{\mathbf{k}}$ and $v_{\mathbf{k}}$, satisfy the linear equations of motion followed from the Hamiltonian $\mathcal{H}_{0}$,

$$
\begin{aligned}
u_{\mathbf{k}}^{\prime \prime}-\frac{2}{\tau} u_{\mathbf{k}}^{\prime}+k^{2} u_{\mathbf{k}} & =0, \\
v_{\mathbf{k}}^{\prime \prime}-\frac{2}{\tau} v_{\mathbf{k}}^{\prime}+k^{2} v_{\mathbf{k}}+\frac{M_{\mathrm{eff}}^{2}}{H^{2} \tau^{2}} v_{\mathbf{k}} & =0,
\end{aligned}
$$

where $\tau$ is the conformal time defined by $\mathrm{d} \tau=\mathrm{d} t / a(t)$, the prime denotes the derivative with respect to $\tau$, and we have approximated the background by a de Sitter spacetime.

The solutions to (18) and (19) are given by linear combinations of the Hankel functions of first and second kind. Requiring that the solutions approach those in the Minkowski positive frequency functions, or those for the Bunch-Davies vacuum [8], in the limit $k \gg a H$,

$$
R u_{\mathbf{k}}, \quad v_{\mathbf{k}} \rightarrow i \frac{H}{\sqrt{2 k}} \tau e^{-i k \tau},
$$

we obtain

$$
u_{\mathbf{k}}=\frac{H}{R \sqrt{2 k^{3}}}(1+i k \tau) e^{-i k \tau}
$$

and

$$
v_{\mathbf{k}}=-i e^{i\left(\nu+\frac{1}{2}\right) \frac{\pi}{2}} \frac{\sqrt{\pi}}{2} H(-\tau)^{3 / 2} H_{\nu}^{(1)}(-k \tau), \quad \text { for } \quad M_{\mathrm{eff}}^{2} / H^{2} \leq 9 / 4
$$

where $\nu=\sqrt{9 / 4-M_{\text {eff }}^{2} / H^{2}}$, or

$$
v_{\mathbf{k}}=-i e^{-\frac{\pi}{2} \mu+i \frac{\pi}{4}} \frac{\sqrt{\pi}}{2} H(-\tau)^{3 / 2} H_{i \mu}^{(1)}(-k \tau), \quad \text { for } \quad M_{\mathrm{eff}}^{2} / H^{2}>9 / 4
$$


where $\mu=\sqrt{M_{\text {eff }}^{2} / H^{2}-9 / 4}$.

Next we consider the interaction terms as perturbations to $\mathcal{H}_{0}$. To calculate the power spectrum up to tree-level diagrams, we just need to consider the $\mathcal{H}_{2}$ term which describes the correlation between the curvature and isocurvature modes,

$$
H_{2}^{I}=\int \mathrm{d}^{3} \mathbf{x} \mathcal{H}_{2}^{I}=-c_{2} a^{3} \int \frac{\mathrm{d}^{3} \mathbf{k}}{(2 \pi)^{3}} \delta \sigma_{\mathbf{k}}^{I} \dot{\delta \theta_{-\mathbf{k}}^{I}} .
$$

The two-point function of $\delta \theta^{2}$ can be calculated by the in-in formulism as

$$
\begin{aligned}
\left\langle\delta \theta^{2}\right\rangle \equiv & \left\langle 0\left|\left[\bar{T} \exp \left(i \int_{t_{0}}^{t} d t^{\prime} H_{I}\left(t^{\prime}\right)\right)\right] \delta \theta_{I}^{2}(t)\left[T \exp \left(-i \int_{t_{0}}^{t} d t^{\prime} H_{I}\left(t^{\prime}\right)\right)\right]\right| 0\right\rangle, \\
= & \langle 0| \bar{T}\left(1+i \int_{t_{0}}^{t} d t^{\prime} H_{I}\left(t^{\prime}\right)-\int_{t_{0}}^{t} d t_{1} \int_{t_{0}}^{t} d t_{2} H_{I}\left(t_{1}\right) H_{I}\left(t_{2}\right)+\ldots\right) \delta \theta_{I}^{2}(t) \\
& \cdot T\left(1+i \int_{t_{0}}^{t} d t^{\prime} H_{I}\left(t^{\prime}\right)-\int_{t_{0}}^{t} d t_{1} \int_{t_{0}}^{t} d t_{2} H_{I}\left(t_{1}\right) H_{I}\left(t_{2}\right)+\ldots\right)|0\rangle, \\
= & \left\langle 0\left|\delta \theta_{I}^{2}\right| 0\right\rangle \\
+ & \int_{t_{0}}^{t} d t_{1} \int_{t_{0}}^{t} d t_{2}\left\langle 0\left|H_{I}\left(t_{1}\right) \delta \theta_{I}^{2} H_{I}\left(t_{2}\right)\right| 0\right\rangle \\
- & 2 \operatorname{Re}\left[\int_{t_{0}}^{t} d t_{1} \int_{t_{0}}^{t_{1}} d t_{2}\left\langle 0\left|\delta \theta_{I}^{2} H_{I}\left(t_{1}\right) H_{I}\left(t_{2}\right)\right| 0\right\rangle\right] \\
+ & \cdots .
\end{aligned}
$$

To calculate the terms sandwiched with the free field vacuum, we use the normal ordering. After that only fully contracted terms survive. The correction from a mediating isocurvature perturbation is depicted in Fig. 2. This gives the correction to the power spectrum of $\delta \theta$ as

$$
(2 \pi)^{3} \delta^{3}\left(\mathbf{p}_{1}+\mathbf{p}_{2}\right) \frac{c_{2}^{2}}{R^{4}} \frac{\mathcal{C}(\nu)}{p_{1}^{3}},
$$

with a factor $\mathcal{C}(\nu)$ which only depends on the effective mass $M_{\text {eff }}$ and rotation speed $\dot{\theta}^{2}$ via $\nu$,

$$
\begin{aligned}
\nu & =\sqrt{\frac{9}{4}-\frac{M_{\mathrm{eff}}^{2}}{H^{2}}}, \quad M_{\mathrm{eff}}^{2}=V_{\sigma \sigma}+3 \dot{\theta}^{2}, \\
\mathcal{C}(\nu) & =\frac{\pi}{8}\left\{\left|\int_{0}^{\infty} d x x^{-1 / 2} H_{\nu}^{(1)}(x) e^{i x}\right|^{2}\right. \\
& \left.-2 \operatorname{Re} \int_{0}^{\infty} d x_{1} x_{1}^{-1 / 2} H_{\nu}^{(1)}\left(x_{1}\right) e^{-i x_{1}} \int_{x_{1}}^{\infty} d x_{2} x_{2}^{-1 / 2}\left(H_{\nu}^{(1)}\left(x_{2}\right)\right)^{*} e^{-i x_{2}}\right\} .
\end{aligned}
$$

Applying the above to Eq. (13), the power spectrum of the curvature perturbation on comoving slices is given by

$$
\mathcal{P}_{\mathcal{R}}=\frac{H^{4}}{4 \pi^{2} R^{2} \dot{\theta}^{2}}\left[1+8 \mathcal{C}(\nu)\left(\frac{\dot{\theta}}{H}\right)^{2}\right] .
$$


The calculation of $\mathcal{C}(\nu)$ for real $\nu$ has been done in [34] by numerical integral. It is our main task to calculate $\mathcal{C}(\nu)$ in the case when $\nu$ is pure imaginary in Section III.

As a warmup, we do the calculation of $\mathcal{C}(\nu)$ for $0<\nu<3 / 2$ analytically in this section. In this case, we first write

$$
\mathcal{C}(\nu)=\frac{\pi}{8}(\mathcal{A}-\mathcal{B})
$$

where $\mathcal{A}$ and $\mathcal{B}$ are the first and second terms, respectively, of Eq. (31), and calculate the integrals $\mathcal{A}$ and $\mathcal{B}$ piece by piece: We use the asymptotic from of the Hankel function at $x \ll 1$ for the integration from 0 to 1 , and the asymptotic from at $x \gg 1$ for the integration from 1 to $\infty$. We can see that, the main contribution to the integral is from $0<x<1$, i.e., the infrared era when the wavelength of the mode has been stretched out of horizon.

The asymptotic behavior of the Hankel function in the ultraviolet limit is in the form of an ordinary wave, but that in the infrared limit needs some caution since there is a spurious discontinuity and divergence at integer $\nu$. To avoid it one should take more terms of asymptotic expansion into account instead of keeping only the leading order term. We defer the detailed discussion in Appendix A. Here we refer to the result given by Eqs. (A1) and (A2),

$$
\begin{aligned}
\mathcal{A} & =\left|\int_{0}^{1} d x x^{-1 / 2} H_{\nu}^{(1)}(x) e^{i x}+\int_{1}^{\infty} d x x^{-1 / 2} H_{\nu}^{(1)}(x) e^{i x}\right|^{2}, \\
\longrightarrow & \sum_{k=0}^{\infty} \frac{(-)^{k}}{2^{2 k} k !}\left\{\frac{1+i \cot \nu \pi}{2^{\nu} \Gamma(k+\nu+1)} \int_{0}^{1} d x x^{2 k+\nu-1 / 2} e^{i x}\right. \\
& \left.-\frac{i 2^{\nu}}{\sin \nu \pi \Gamma(k-\nu+1)} \int_{0}^{1} d x x^{2 k-\nu-1 / 2} e^{i x}\right\}+\left.\int_{1}^{\infty} d x \sqrt{\frac{2}{\pi}} \frac{e^{i(2 x-\nu \pi / 2-\pi / 4)}}{x}\right|^{2}, \\
= & \mid \sum_{k=0}^{\infty} \frac{(-)^{k}}{2^{2 k} k !}\left\{\frac{1+i \cot \nu \pi}{2^{\nu} \Gamma(k+\nu+1)} i^{2 k+\nu+1 / 2} \gamma(2 k+\nu+1 / 2,-i)\right. \\
& \left.-\frac{i^{2 k-\nu+3 / 2} 2^{\nu}}{\sin \nu \pi \Gamma(k-\nu+1)} \gamma(2 k-\nu+1 / 2,-i)\right\}+\left.\sqrt{\frac{2}{\pi}} e^{-i \frac{\pi}{2}\left(\nu+\frac{1}{2}\right)}\left(\pi+i \mathrm{E}_{1}(2 i)\right)\right|^{2},
\end{aligned}
$$

where some of the integrals are expressed in terms of the incomplete gamma function,

$$
\gamma(s, p)=\int_{0}^{p} t^{s-1} e^{-t} d t
$$

The incomplete gamma function is defined originally on the real $s$ axis, but can be analytically continued to the complex plane with a branch cut from $-\infty$ to 0 . For a given wavelength, the third term in (34) is the integral from the ultraviolet era $x>1$, which contributes little to the final result.

The evaluation of $\mathcal{B}$ is a little tricky since there is an overall time ordering operator. 
Keeping this in mind, we split the integrals on two axes as

$$
\begin{aligned}
\mathcal{B} & =2 \operatorname{Re} \int_{0}^{1} d x_{1} x_{1}^{-1 / 2} H_{\nu}^{(1)}\left(x_{1}\right) e^{-i x} \int_{x_{1}}^{1} d x_{2} x_{2}^{-1 / 2} H_{\nu}^{(2)}\left(x_{2}\right) e^{-i x_{2}} \\
& +2 \operatorname{Re} \int_{0}^{1} d x_{1} x_{1}^{-1 / 2} H_{\nu}^{(1)}\left(x_{1}\right) e^{-i x} \int_{1}^{\infty} d x_{2} x_{2}^{-1 / 2} H_{\nu}^{(2)}\left(x_{2}\right) e^{-i x_{2}} \\
& +2 \operatorname{Re} \int_{1}^{\infty} d x_{1} x_{1}^{-1 / 2} H_{\nu}^{(1)}\left(x_{1}\right) e^{-i x} \int_{x_{1}}^{\infty} d x_{2} x_{2}^{-1 / 2} H_{\nu}^{(2)}\left(x_{2}\right) e^{-i x_{2}}
\end{aligned}
$$

As before, the second line (37) can be neglected since it is small compared to the first line (36), while the third line (38) can be discarded directly if one adds a small imaginary part $i \epsilon$ to $x$ and do the integral in the ultraviolet limit. Now the double integral in the first line (36) is equivalent to the integration over the upper triangle bounded by the $x_{2}$-axis, $x_{2}=1$ and $x_{1}=x_{2}$. Since the integrand is in the form $2 f\left(x_{1}\right) f\left(x_{2}\right)$, it is in particular symmetric with respect to the interchange of $x_{1}$ and $x_{2}$. Hence the integral is equal to a half of that over the square bounded by $x_{1}=x_{2}=0$ and $x_{1}=x_{2}=1$. The result is then given by the square of the integral $\int_{0}^{1} d x f(x)$, that is,

$$
\begin{aligned}
\mathcal{B} \longrightarrow & \operatorname{Re}\left[\int_{0}^{1} d x x^{1 / 2} Y_{\nu}(x) e^{-i x}\right]^{2}, \\
= & \operatorname{Re}\left[\sum _ { k = 0 } ^ { \infty } \frac { ( - ) ^ { k } } { 2 ^ { 2 k } k ! } \left(\frac{\cot \nu \pi}{2^{\nu} \Gamma(k+\nu+1)} \int_{0}^{1} d x x^{2 k+\nu-1 / 2} e^{-i x}\right.\right. \\
& \left.\left.-\frac{2^{\nu}}{\sin \nu \pi \Gamma(k-\nu+1)} \int_{0}^{1} d x x^{2 k-\nu-1 / 2} e^{-i x}\right)\right]^{2}, \\
= & \operatorname{Re}\left[\sum _ { k = 0 } ^ { \infty } \frac { ( - ) ^ { k } } { 2 ^ { 2 k } k ! } \left(\frac{(-i)^{2 k+\nu+1 / 2} \cot \nu \pi}{2^{\nu} \Gamma(k+\nu+1)} \Gamma(2 k+\nu+1 / 2,0, i)\right.\right. \\
& \left.\left.-\frac{2^{\nu}(-i)^{2 k-\nu+1 / 2}}{\sin \nu \pi \Gamma(k-\nu+1)} \Gamma(2 k-\nu+1 / 2,0, i)\right)\right]^{2} .
\end{aligned}
$$

In summary, we obtain an approximate analytical expression for $\mathcal{C}(\nu)$ by combining (33), (34) and (39). It is depicted in Fig. 3 in which the sum over $k$ is truncated at $N=10^{3}$. We see that our approximate formula reproduces the one obtained numerically in [34] fairly well. In fact, as discussed in detail in Appendix $\mathrm{A}$, the convergence of this series is very fast and a truncation at $N=3$ is found to give a result which is almost indistinguishable from that at $N=10^{3}$.

\section{HEAVY ISOCURVATURE MODES: INFLATION UNDER EFFECTIVE FIELD THEORY}

For an isocurvature mode which has a large effective mass, that is, $M_{\text {eff }} / H>3 / 2$, the parameter $\nu$ becomes pure imaginary. Therefore we introduce a real parameter $\mu$ by

$$
\nu \equiv i \mu=i \sqrt{\left(\frac{M_{\mathrm{eff}}}{H}\right)^{2}-\frac{9}{4}}
$$




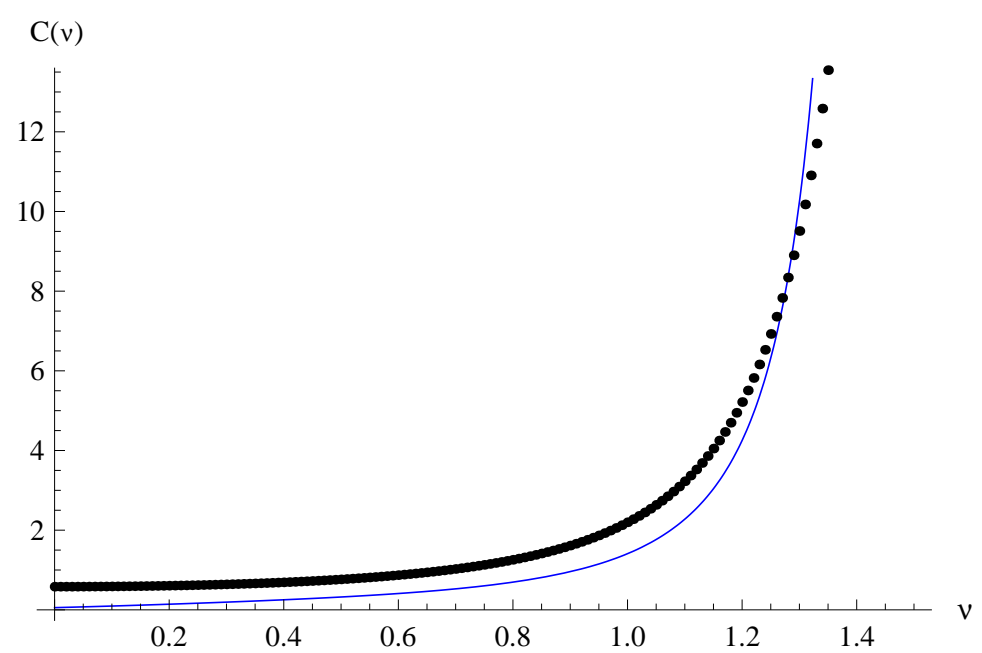

FIG. 3: The blue curve depicts an approximate analytic expression for $\mathcal{C}(\nu)$ given by Eqs. (34) and (39), discarding the contributions coming from the ultraviolet part $x>1$. The dots are those read from the numerical result obtained in [34]. The summation over $k$ in these equations is truncated at $k \leq N=10^{3}$.

Then the correction to the power spectrum is written as

$$
\begin{aligned}
\mathcal{C}(\mu) & \equiv \frac{\pi}{8} e^{-\mu \pi}(\alpha-\beta) \\
\alpha & =\left|\int_{0}^{\infty} d x x^{-1 / 2} H_{i \mu}^{(1)}(x) e^{i x}\right|^{2}, \\
\beta & =2 \operatorname{Re} \int_{0}^{\infty} d x_{1} x_{1}^{-1 / 2} H_{i \mu}^{(1)}\left(x_{1}\right) e^{-i x_{1}} \int_{x_{1}}^{\infty} d x_{2} x_{2}^{-1 / 2}\left(H_{i \mu}^{(1)}\left(x_{2}\right)\right)^{*} e^{-i x_{2}} .
\end{aligned}
$$

Note the existence of an overall exponential suppression factor $e^{-\mu \pi}$. This kills almost all the contributions in $\alpha$ and $\beta$ except for the one which contains an exponential enhancement factor that exactly cancels it. The above can be evaluated numerically as depicted in Fig. 4. But we can evaluate it analytically in the limit when the isocurvaton is very heavy, i.e. $\mu \gg 1$, which is the case when one expects an effective single-field description holds.

First we consider $\alpha$. The integral in $\alpha$ is

$$
\int_{0}^{\infty} d x x^{1 / 2} H_{i \mu}^{(1)}(x) e^{i x}=\int_{0}^{\infty} d x x^{1 / 2} J_{i \mu}(x) e^{i x}+i \int_{0}^{\infty} d x x^{1 / 2} N_{i \mu}(x) e^{i x} .
$$

The first term on the right hand side can be integrated directly as

$$
\begin{aligned}
\int_{0}^{\infty} d x x^{1 / 2} J_{i \mu}(x) e^{i x} & =\frac{\Gamma(1 / 2+i \mu)}{2^{i \mu}(-i)^{1 / 2+i \mu} \Gamma(1+i \mu)}{ }_{2} F_{1}\left(\frac{1}{4}+\frac{i \mu}{2}, \frac{3}{4}+\frac{i \mu}{2} ; 1+i \mu ; 1\right) \\
& =\frac{e^{i \pi / 4}}{\sqrt{2 \pi}} e^{-\mu \pi / 2}
\end{aligned}
$$

In obtaining this we have used the special value of hypergeometric function when the last argument is 1, see (B5) in Appendix $\mathrm{B}$, and relation of gamma function. Next, we can calculate the second integral in (44). There is a possible spurious divergence if we integrate 
it directly. Let us add a small positive imaginary part $i \epsilon$ to the exponent to make it converge, i.e. to write the exponent as $e^{i x-\epsilon x}$. Therefore, the integral is

$$
\begin{aligned}
& \int_{0}^{\infty} d x x^{1 / 2} N_{i \mu}(x) e^{i x} \\
= & \frac{\cot i \mu \pi \Gamma(1 / 2+i \mu)}{2^{i \mu} \Gamma(1+i \mu)(-2 i \epsilon)^{1 / 4+i \mu / 2}}{ }_{2} F_{1}\left(\frac{1}{4}+\frac{i \mu}{2}, \frac{1}{4}+\frac{i \mu}{2} ; 1+i \mu ; \frac{1}{-2 i \epsilon}\right) \\
- & \frac{2^{i \mu} \Gamma(1-i \mu)}{\sin i \mu \pi \Gamma(1 / 2-i \mu)(-2 i \epsilon)^{1 / 4-i \mu / 2}}{ }_{2} F_{1}\left(\frac{1}{4}-\frac{i \mu}{2}, \frac{1}{4}-\frac{i \mu}{2} ; 1-i \mu ; \frac{1}{-2 i \epsilon}\right) .
\end{aligned}
$$

Then this is the situation when the last argument of the Gaussian hypergeometric function is large, and (B6) can be applied to connect it to

$$
\begin{aligned}
\text { (47) }) & =\frac{\operatorname{coth} \mu \pi \Gamma\left(\frac{1}{2}+i \mu\right)}{2^{i \mu} \Gamma(1+i \mu)} \frac{2 \Gamma(1+i \mu)}{\Gamma\left(\frac{1}{4}+\frac{i \mu}{2}\right) \Gamma\left(\frac{3}{4}+\frac{i \mu}{2}\right)}{ }_{2} F_{1}\left(\frac{1}{4}+\frac{i \mu}{2}, \frac{1}{4}+\frac{i \mu}{2} ; 1+i \mu ;-2 i \epsilon\right) \\
& -\frac{2^{i \mu} \Gamma\left(\frac{1}{2}-i \mu\right)}{i \sinh \mu \pi \Gamma(1-i \mu)} \frac{2 \Gamma\left(\frac{1}{2}-i \mu\right)}{\Gamma\left(\frac{1}{4}-\frac{i \mu}{2}\right) \Gamma\left(\frac{3}{4}-\frac{i \mu}{2}\right)}{ }_{2} F_{1}\left(\frac{1}{4}-\frac{i \mu}{2}, \frac{1}{4}-\frac{i \mu}{2} ; 1-i \mu ;-2 i \epsilon\right) .
\end{aligned}
$$

Now the divergence disappears, and we can take $\epsilon \rightarrow 0$ and use (B10) to get

$$
\text { (47) }=\frac{2 \operatorname{coth} \mu \pi \Gamma\left(\frac{1}{2}+i \mu\right)}{2^{i \mu} \Gamma\left(\frac{1}{4}+\frac{i \mu}{2}\right) \Gamma\left(\frac{3}{4}+\frac{i \mu}{2}\right)}-\frac{2^{1+i \mu} \Gamma\left(\frac{1}{2}-i \mu\right)}{i \sinh \mu \pi \Gamma\left(\frac{1}{4}-\frac{i \mu}{2}\right) \Gamma\left(\frac{3}{4}-\frac{i \mu}{2}\right)} \text {. }
$$

Next we take (B1) to cancel these gamma functions. We are left with

$$
\text { (48) }=\sqrt{\frac{2}{\pi}}\left(\operatorname{coth} \mu \pi+\frac{i}{\sinh \mu \pi}\right) \text {. }
$$

Therefore we have that

$$
\alpha=\frac{1}{\pi}\left|\frac{e^{\mu \pi / 2}}{2}-\frac{\sqrt{2}}{\sinh \mu \pi}+i\left(\frac{e^{-\mu \pi}}{2}+\sqrt{2} \operatorname{coth} \mu \pi\right)\right|^{2} .
$$

This is an exact result, with no approximations. We see that when the effective isocurvature mass is large, this goes as

$$
\alpha \rightarrow \frac{2}{\pi} \operatorname{coth}^{2} \mu \pi \sim 1
$$

Together with the factor $e^{-\mu \pi}$ in (41), we now that the contribution from $\alpha$ is exponentially suppressed.

Now we move to the evaluation of $\beta$,

$$
\beta=2 \operatorname{Re} \int_{0}^{\infty} d x_{1} x_{1}^{-1 / 2} H_{i \mu}^{(1)}\left(x_{1}\right) e^{-i x_{1}} \int_{x_{1}}^{\infty} d x_{2} x_{2}^{-1 / 2}\left(H_{i \mu}^{(1)}\left(x_{2}\right)\right)^{*} e^{-i x_{2}} .
$$


Take the asymptotic behavior (C5) we have got in Appendix C, we can see

$$
\begin{aligned}
\beta= & \frac{4}{\pi} \frac{e^{\pi \mu}}{\mu} \operatorname{Re} \int_{0}^{\infty} d x_{1} x_{1}^{i \mu-1 / 2} \exp \left[-\frac{x_{1}^{2}}{4 \mu} e^{-i \pi / 4}-i x_{1}\right] \\
& \times \int_{x_{1}}^{\infty} d x_{2} x_{2}^{-i \mu-1 / 2} \exp \left[-\frac{x_{2}^{2}}{4 \mu} e^{i \pi / 4}-i x_{2}\right]
\end{aligned}
$$

which contains a double integral. We first deal with the integral of $x_{2}$. As we have done in calculating $\alpha$, we can take the Taylor series of the exponent for $x_{2}$ and the integral of $x_{2}$ can be done term by term to get

$$
\begin{aligned}
& \int_{x_{1}}^{y_{1}} d x_{2} x_{2}^{-i \mu-1 / 2} \exp \left[-\frac{x_{2}^{2}}{4 \mu} e^{i \pi / 4}-i x_{2}\right] \\
= & \sum_{k=0}^{\infty} \frac{(-)^{k}}{k !} \int_{x_{1}}^{y_{1}} d x_{2} x_{2}^{-i \mu-1 / 2}\left(\frac{x_{2}^{2}}{4 \mu} e^{i \pi / 4}+i x_{2}\right)^{k}, \\
= & \sum_{k=0}^{\infty} \frac{(-)^{k}}{k !\left(k+\frac{1}{2}-i \mu\right)^{4 \mu}} \frac{1}{4 \mu} \\
\times & \left\{x_{1}^{k+1 / 2-i \mu}\left(i+\frac{(-)^{1 / 4} x_{1}}{4 \mu}\right)^{k}\left[(-)^{3 / 4} x_{1}-4 \mu\right]_{2} F_{1}\left(1, \frac{3}{2}+2 k-i \mu ; \frac{3}{2}+k-i \mu ; \frac{(-)^{3 / 4} x_{1}}{4 \mu}\right)\right. \\
- & \left.y_{1}^{k+1 / 2-i \mu}\left(i+\frac{(-)^{1 / 4} y_{1}}{4 \mu}\right)^{k}\left[(-)^{3 / 4} y_{1}-4 \mu\right]_{2} F_{1}\left(1, \frac{3}{2}+2 k-i \mu ; \frac{3}{2}+k-i \mu ; \frac{(-)^{3 / 4} y_{1}}{4 \mu}\right)\right\}
\end{aligned}
$$

Again, integrating $x_{2}$ from 0 to $\infty$ in (54) will give us a summation of infinities, which is unphysical since we know that in (53) there is an exponential suppression which will converge the integral. Thus, we take a UV cutoff at $y_{1}$, and set $y_{1} \rightarrow \infty$ after all the integrations and summations are done. Thus this integral can be expressed by its lower bound $x_{1}$ and upper bound $y_{1}$ as above via the hypergeometric function ${ }_{2} F_{1}$. We make use of the asymptotic behavior of this function when $\mu \gg 1$ in Appendix $B$. From (B9), we see that when $\mu$ is large and $k \ll \mu$, the hypergeometric functions with different $k$ 's are the same, and we can resum the polynomials to get back to an exponent, which yields

$$
\text { (⒌5) }=\frac{i}{\mu}\left\{y_{1}^{1 / 2-i \mu} \exp \left[-y_{1}\left(i+\frac{(-)^{1 / 4} y_{1}}{4 \mu}\right)\right]-x_{1}^{1 / 2-i \mu} \exp \left[-x_{1}\left(i+\frac{(-)^{1 / 4} x_{1}}{4 \mu}\right)\right]\right\} \text {. }
$$

Now we can take the limit of integration upper bound $y_{1}$ to be infinite. This kills the first term in (56). Then, substituting (56) into (52),

$$
\beta=-\frac{4}{\pi} \frac{e^{\pi \mu}}{\mu^{2}} \operatorname{Re} i \int_{0}^{\infty} d x_{1} \exp \left[-\frac{\sqrt{2} x_{1}^{2}}{4 \mu}-2 i x_{1}\right]
$$

This integral can be expressed by the error function,

$$
\operatorname{erf}(z)=\frac{2}{\sqrt{\pi}} \int_{0}^{z} e^{-t^{2}} d t
$$




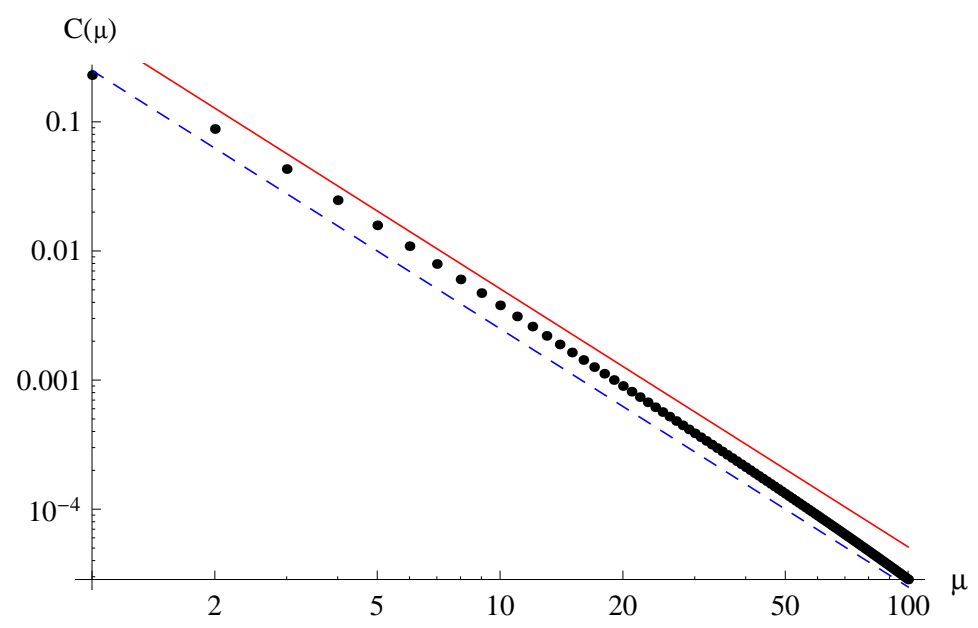

FIG. 4: The red curve shows $\mathcal{C}(\mu)$ given by our analytical approximation (60), which is also the one derived from the effective single-field approach (62). The black dots are the result of numerical integrations of (41). There is a small deviation when $\mu \sim \mathcal{O}(10)$

as

$$
\beta=2^{7 / 4} \frac{e^{\mu \pi}}{\sqrt{\pi \mu^{3}}} e^{-2 \sqrt{2} \mu} \operatorname{Re}\left[i \operatorname{erf}\left(i 2^{3 / 4} \mu\right)\right] .
$$

We can see that it depends on a power-law series of $\mu$ when $\mu$ is large. To be specific, let us can take the Taylor series of $\beta$ around infinity to get

$$
\beta=-2 \frac{e^{\mu \pi}}{\pi \mu^{2}}\left[1+\sum_{k=1}^{\infty} \frac{(2 k-1) ! !}{2^{5 k / 2} \mu^{k}}\right] .
$$

This is the only term that contributes to the final correction to the power spectrum, and we see that the leading term is proportional to $\mu^{-2}$ (thus to $M_{\text {eff }}^{2}$ ) when the effective mass of the isocurvature mode is large. We take this leading term and go back from the combination of (32), (41) and (59) to obtain our final result

$$
\begin{aligned}
\mathcal{C}(\mu) & \approx \frac{1}{4 \mu^{2}} \\
\mathcal{P}_{\mathcal{R}} & \approx \mathcal{P}_{\mathcal{R}}^{(0)}\left[1+2 \frac{H^{2}}{M_{\mathrm{eff}}^{2}}\left(\frac{\dot{\theta}}{H}\right)^{2}\right] .
\end{aligned}
$$

As before $\approx$ denotes neglecting the terms of higher power. Note the dependence on $\mu$. It is not an exponential suppression, but a power-law one. We reached (61) under the approximation that $\mu$ is large as well as $\dot{\theta} / H$ is small. As depicted in Fig. 4, the analytic approximation (60) reproduces a numerical evaluation of the formula (41) well. This is our main result.

To compare this with the one obtained by the effective-single-field approach, let us refer to the result in [36, 38, 40, 41] where the two-field case with large isocurvature mass is considered. After integrating out the heavy field, the power spectrum is corrected in terms 
of the effective sound speed as

$$
c_{s}^{-2}=1+\frac{4 H^{2}}{\tilde{M}_{\mathrm{eff}}^{2}}\left(\frac{\dot{\theta}}{H}\right)^{2},
$$

where to leading order $\tilde{M}_{\text {eff }}^{2}=V_{\sigma \sigma}-\dot{\theta}^{2}$ in our notation. We first note that the definition of effective mass $\tilde{M}_{\text {eff }}^{2}$ is different from ours by $4 \dot{\theta}^{2}$, which is not important since it is assumed to be small when the perturbative in-in formulism can be applied. Besides, we also see that our result (60), which gives the power spectrum a correction factor corresponding to $c_{s}^{-1}$, is exactly the same as (62) at leading order when $\dot{\theta}^{2}$ is small. So we have shown the equivalence of both methods applying on the same model.

\section{CONCLUSIONS AND DISCUSSIONS}

In this paper, we studied a two-field model of inflation in which the curvature mode, that is the mode along the inflationary trajectory, is light while the isocurvature mode, that is perpendicular to the trajectory, is heavy. To simplify the problem, we focused on a situation in which the field is in a circular motion with a small constant angular velocity. By computing a process that describes the effect of the heavy isocurvature mode on the curvature perturbation 2-point function by the in-in formalism, we derived a correction to the curvature perturbation power spectrum due to the heavy mode intermediation. The result tells us that the correction is proportional to $\left(\dot{\theta} / M_{\text {eff }}\right)^{2}$, which is exactly the same as the one obtained by the effective single-field approach by integrating out the heavy field.

Here let us reconsider our assumptions, and discuss the validity and limitaions of both methods. We already know that only if

$$
\frac{\dot{\theta}^{2}}{H^{2}} \ll 1
$$

one can use perturbation theory in the in-in formalism which we employed. On the other hand, in the case of a sharp turn with large angular velocity, even for a very short time, our method fails. It is demonstrated in [39, 45] that such a case cannot be described by an effective single-field action either. The other assumption is $M_{\text {eff }}^{2} \gg H^{2}$ which we use to get our final analytic expressions. Although this is not necessary for the use of the in-in formulism, it enabled us to obtain a simple analytic result and to easily compare this with the effective single-field approach in which the heavy modes are integrated out.

Some papers discussed the requirements of effective single field theory to hold besides the isocurvature mode is heavy. The main constraint is called the adiabatic condition [40],

$$
\left|\frac{\mathrm{d}}{\mathrm{d} t} \log \dot{\theta}\right| \ll M_{\mathrm{eff}}, \quad \text { or } \quad\left|\frac{\mathrm{d}}{\mathrm{d} t} \log \left(c_{s}^{-2}-1\right)\right| \ll M_{\mathrm{eff}} .
$$

In our constant turn case these conditions are satisfied since we have $\ddot{\theta}=0$. It is shown that the decoupling of the heavy field by integrating it out is valid only when slow-roll condition is preserved [42]. In [39] the authors claim that to keep slow-roll in the classical trajectory one should impose

$$
\frac{V^{\prime \prime}}{H} \gg \frac{\dot{\theta}}{H}
$$




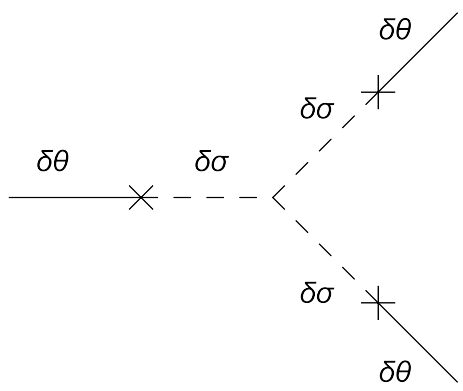

FIG. 5: This is the main contribution to large non-Gaussianity evaluated in [34].
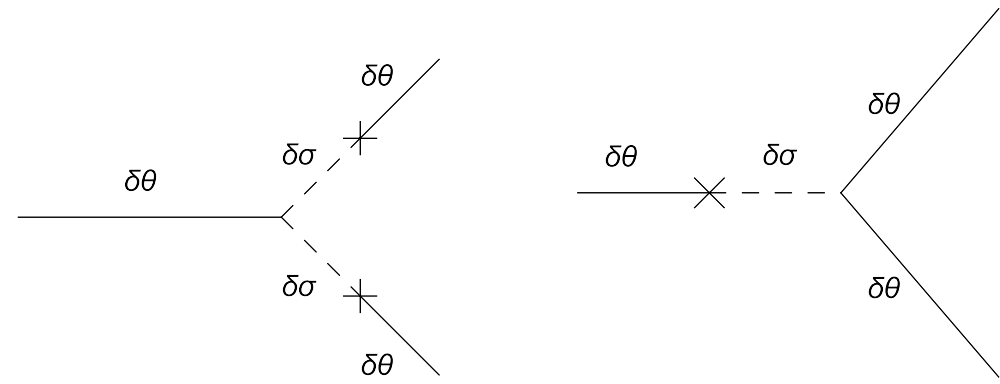

FIG. 6: These are other possible subleading contributions to the non-Gaussianity in quasi-single field inflation.

which is satisfied for $\mu \gg 1$. On the other hand, for $\nu \rightarrow 3 / 2$ which corresponds to the case when not only the inflaton but also the isocurvaton have their masses negligible, there does not exist even an appropriate classical quasi-single-field trajectory. This is probably the reason why we see the divergent behavior in Fig. 3 at $\nu \rightarrow 3 / 2$. In this case, one should abandon the effective single-field description, and turn to other methods used in the standard slow-roll multi-field case as mentioned in Section [1]

It is important to study the implications of quasi-single field models in realistic situations. It surely represents a kind of turning process, but it must be adiabatic. If the trajectory is straight before and after the adiabatic turning, the power spectrum (61) is only modified during the turning process, which implies a feature at a characteristic scale determined by the time-dependence of $\dot{\theta}$. It will be interesting to study this "adiabatic turn" quasi-single field inflation in more detail. For instance, we know this scale-dependence can only be detected if the turning occurs not too late at the inflationary stage. In hybrid inflation [27], inflation ends by a sharp turn in the field space and the mode that was isocurvature during inflation turns into the waterfall field and becomes important [28 32]. In particular, recently it was pointed out that a spiky feature may appear in the spectrum and bispectrum for a certain parameter range of hybrid inflation [33]. The waterfall process is not adiabatic. Thus our method cannot be applied. Nevertheless, it is interesting to see if an extension of the present method based on the in-in formalism can shed more light on these cases of hybrid inflation.

Finally, let us comment on possible developments from our current work. We notice that our result (61) is exact only at leading order in the limit $\mu \rightarrow \infty$. Beyond this limit we can calculate higher order correction in $1 / M_{\text {eff }}$ to compensate possible deviations from the leading order result. An interesting possibility emerges when we consider next-to-leading order terms. We know in the EFT approach, corrections to the power spectrum from the isocurvaton come in only via $V_{\sigma \sigma}$, which means the next-to-leading order correction should be of order $1 / V_{\sigma \sigma}^{2} \sim 1 / \tilde{M}_{\text {eff }}^{4}$. On the other hand, in our approach, the effect comes from $\mu=\sqrt{M_{\text {eff }}^{2} / H^{2}-9 / 4} \sim M_{\text {eff }}$, thus naively the next-to-leading correction will be $1 / M_{\text {eff }}^{3}$. Therefore, unless we has a mechanism to cancel all the contributions from the terms with odd power indices, we will face this inconsistency. We need further investigations to find out the possible implications behind this fact.

Another access to check this consistency is to consider the higher order correlations, specifically the 3-point function of the curvature perturbation. Actually, one of the main motivations for developing the quasi-single field approach was to calculate the 
non-Gaussianity expected in such a model [34, 43]. It is found that only the vertex given by $V^{\prime \prime \prime}$, i.e., the last term in (11) corresponding to the diagram shown in Fig. 5 was of most importance in contributing to large non-Gaussianities. This is because the self-interaction of the isocurvature mode, i.e. $V^{\prime \prime \prime}$, is possible to be large, while the other vertices in (11), as are depicted in Fig. 6, will give a negligible contribution. Under the same assumptions, we can also calculate the similar coefficient $s(\nu)$ and $\alpha(\nu)$ of non-Gaussian parameter $f_{\mathrm{NL}}$ in the three-point correlations which also depend on the effective mass, extrapolate it to the case with a large isocurvaton mass, and compare it with the non-Gaussianity in effective

field approach [36, 38]. It is very interesting to see whether these two results still coincides with each other, and if so, whether we can prove the equivalence of the two approaches to all orders.

Note: When this work was nearly completed, we were acquainted by private communication that a related work [44] on this issue was also approaching its completion. We thank the authors of [44] for synchronizing our submission to the arXiv.

\section{Acknowledgments}

We are grateful to Yifu Cai for useful discussions on numerical methods. SP thanks the hospitality during his visit to Yukawa Institute for Theoretical Physics for the workshop "2012 Asia Pacific School/Workshop on Cosmology and Gravitation" (YITP-W-11-26) when this work was initiated. SP also thanks Bin Chen, Qiang Xu, Jiaju Zhang for valuable comments on the work. We thank the referee and editor of JCAP, who have pointed out the inconsistency between our result in the first version and EFT's, and give us suggestions on how to improve the accuracy in our calculation. This work is supported in part by the JSPS Grant-in-Aid for Scientific Research (A) No. 21244033, and by the MEXT Grant-inAid for the global COE program at Kyoto University, "The Next Generation of Physics, Spun from Universality and Emergence". SP is supported by the NSFC Grant No.10975005 and Scholarship Award for Excellent Doctoral Student granted by Ministry of Education of China.

\section{Appendix A: The Asymptotic Form of Hankel Functions of Real Order}

Here we give several useful expressions for the asymptotic expansion of the Hankel functions, paying particular attention to the case of integer orders

We start from the series definition of the Bessel function,

$$
J_{\nu}(x) \longrightarrow \sum_{k=0}^{\infty} \frac{(-)^{k}}{k !} \frac{1}{\Gamma(k+\nu+1)}\left(\frac{x}{2}\right)^{2 k+\nu}
$$

From this definition the Neumann function is defined by

$$
\begin{aligned}
Y_{\nu}(x) & =\cot \nu \pi J_{\nu}(x)-\frac{1}{\sin \nu \pi} J_{-\nu}(x) \\
& =\sum_{k=0}^{\infty} \frac{(-)^{k}}{k !}\left(\frac{x}{2}\right)^{2 k}\left[\frac{\cot \nu \pi}{\Gamma(k+\nu+1)}\left(\frac{x}{2}\right)^{\nu}-\frac{1}{\sin \nu \pi \Gamma(k-\nu+1)}\left(\frac{x}{2}\right)^{-\nu}\right] .
\end{aligned}
$$


For $\nu>0$, it seems that we can neglect the first term in (A2) since it is small compared to the second one when $x \rightarrow 0$. However, both terms suffer from divergence when $\nu$ is an integer, hence the first term cannot be simply discarded.

To see how the divergence in each term is canceled with each other, let us consider first the behavior at $\nu=0$. In this limit, we have

$$
\begin{aligned}
\lim _{\nu \rightarrow 0} Y_{\nu}(x)= & \frac{1}{\nu \pi}\left[1+\nu \ln \left(\frac{x}{2}\right)\right]-\frac{1}{\nu \pi}\left[1-\nu \ln \left(\frac{x}{2}\right)\right] \\
& +\sum_{k=1}^{\infty} \frac{(-)^{k}}{k !}\left\{\frac{1}{\nu \pi \Gamma(k+1)}\left[1+\nu \ln \left(\frac{x}{2}\right)\right]-\frac{1}{\nu \pi \Gamma(k+1)}\left[1-\nu \ln \left(\frac{x}{2}\right)\right]\right\} \\
= & \frac{2}{\pi} J_{0}(x) \ln \frac{x}{2} \\
\longrightarrow & \frac{2}{\pi} \ln \frac{x}{2} \text { for } x \rightarrow 0 .
\end{aligned}
$$

Thus we obtain a finite result because of the cancelation of $1 / \nu$ divergences coming from both terms.

Now consider the case when $\nu$ is an integer. In our case, since $\nu \leq 3 / 2$, only the case $\nu=1$ concerns us. Introducing $\epsilon \equiv 1-\nu$, we have

$$
\begin{aligned}
\lim _{\nu \rightarrow 1} Y_{\nu}(x)= & \sum_{k=1}^{\infty} \frac{(-)^{k}}{k !}\left(\frac{x}{2}\right)^{2 k}\left[-\frac{1}{\epsilon \pi \Gamma(k+2)}\left(\frac{x}{2}\right)\left(1-\epsilon \ln \left(\frac{x}{2}\right)\right)\right. \\
& \left.-\frac{1}{\epsilon \pi \Gamma(k+\epsilon)}\left(\frac{x}{2}\right)^{-1}\left(1+\epsilon \ln \left(\frac{x}{2}\right)\right)\right] \\
= & -\frac{2}{\pi x}+\frac{2}{\pi} J_{1}(x) \ln \left(\frac{x}{2}\right) \\
\longrightarrow & -\frac{2}{\pi x} \text { when } x \rightarrow 0 .
\end{aligned}
$$

Thus there is no divergence as $1 /(1-\nu)$.

Something subtle appears when we want to truncate the summation (A2) to finite terms. From the calculation above, we see that each term in the series is divergent, although the residue is suppressed exponentially by $1 / \Gamma(k+1) \Gamma(k+\nu+1)$ or $1 / \Gamma(k+1) \Gamma(k-\nu+1)$ when $k$ is large. Expanding (A2), we find that for an integer $\nu$, the divergence of the $k^{+}$-th term of positive series, $x^{2 k^{+}+\nu}$, is canceled by the divergence of the $k^{-}$-th term of the negative series, $x^{2 k^{-}-\nu}$, where $k^{-}=k^{+}+\nu$. Truncating the $k^{+}$and $k^{-}$series simultaneously at the same integer $N$ will cancel the divergence at $\nu=0$, but all the other divergences at $\nu \geq 1$ survive. Similarly, one can also truncated at $k^{+}=N$ and $k^{-}=N+m$, where $m$ is a positive integer to cancel the divergence at $\nu=m$, but divergences at all the other integers remain. Thus unless we take the infinite series limit $N \rightarrow \infty$, all the divergences at all the integers cannot be canceled simultaneously.

Fortunately, however, the residues of these divergences are suppressed by $\Gamma(N)$ for large $N$. So in practical contexts this will not cause a problem. For example, if we truncate the series at $k^{+}=k^{-}=1000$ to cancel the divergence at $\nu=0$, the one at $\nu=1$ remains. However since the residue is suppressed by $1 / \Gamma(1000)^{2}$, the divergence becomes effectively invisible unless we take the value of $\nu$ exponentially close to $\nu=1$, within the width of $\Delta \nu \sim 1 / \Gamma(1000)^{2}$. This enables us to draw our analytic curve practically smooth as depicted in Fig. 3 . 
With the above understanding, we obtain the Hankel functions in the form,

$$
\begin{aligned}
& H^{(1)}(x)=J_{\nu}(x)+i Y_{\nu}(x) \rightarrow i Y_{\nu}(x) \\
& H^{(2)}(x)=J_{\nu}(x)-i Y_{\nu}(x) \rightarrow-i Y_{\nu}(x)
\end{aligned}
$$

where the Neumann function is given by the form (A2). The last arrows above hold only for a real $\nu$ but not for an imaginary $\nu, \nu=i \mu$ for $\mu>0$.

As for the ultraviolet behavior at $x \gg 1$, it is trivial,

$$
\begin{aligned}
& H_{\nu}^{(1)} \approx \sqrt{\frac{2}{\pi x}} e^{i(x-\nu \pi / 2-\pi / 4)}, \\
& H_{\nu}^{(2)} \approx \sqrt{\frac{2}{\pi x}} e^{-i(x-\nu \pi / 2-\pi / 4)}, \\
& H_{i \mu}^{(1)} \approx \sqrt{\frac{2}{\pi x}} e^{\mu \pi / 2} e^{i(x-\pi / 4)}, \\
& H_{i \mu}^{(2)} \approx \sqrt{\frac{2}{\pi x}} e^{-\mu \pi / 2} e^{-i(x-\pi / 4)} .
\end{aligned}
$$

These formulae hold for any complex order $\nu$.

\section{Appendix B: Gamma Function and Hypergeometric Function}

A useful relation which is used in the context is the product of two gamma functions whose arguments vary by $1 / 2$ :

$$
\Gamma(z) \Gamma\left(\frac{1}{2}+z\right)=\frac{2 \sqrt{\pi}}{2^{2 z}} \Gamma(2 z) .
$$

Next let us refer to the asymptotic expansion of the Gamma function $\Gamma(z)$ at $z \rightarrow \infty$ on complex plane,

$$
\Gamma(z) \approx(z)^{z-1 / 2} e^{-z \sqrt{2 \pi}}\left\{1+\frac{1}{12 z}-\frac{1}{288 z^{2}}+\ldots\right\} .
$$

In this paper the most commonly used argument is like $a+i \mu$ where $\mu \gg 1$ and $a \ll \mu$. Substituting it into (B2) we have

$$
\Gamma(a \pm i \mu) \approx \sqrt{2 \pi} e^{ \pm i[\mu \ln \mu-\mu+\pi(a-1) / 4]} \mu^{a-1 / 2} e^{\pi \mu / 2} .
$$

Here for simplicity we only preserve the leading term.

The Gaussian hypergeometric function ${ }_{2} F_{1}(a, b ; c ; z)$ appears when dealing with the integrals in section III,

$$
{ }_{2} F_{1}(a, b ; c ; z) \equiv \sum_{n=0}^{\infty} \frac{\Gamma(a+n) \Gamma(b+n) \Gamma(c)}{\Gamma(a) \Gamma(b) \Gamma(c+n)} \frac{z^{n}}{n !} .
$$

We will use the hypergeometric function in three different limit of $z$. First, from the definition, we have

$$
{ }_{2} F_{1}(\alpha, \beta ; \gamma ; 1)=\frac{\Gamma(\gamma) \Gamma(\gamma-\alpha-\beta)}{\Gamma(\gamma-\alpha) \Gamma(\gamma-\beta)}
$$


Now deal with large $z$, which can be connected with the hypergeometric function with a small argument $1 / z$ as

$$
\begin{aligned}
\frac{\Gamma(\alpha) \Gamma(\beta)}{\Gamma(\gamma)}{ }_{2} F_{1}(\alpha, \beta ; \gamma ; z) & =\frac{\Gamma(\alpha) \Gamma(\beta-\alpha)}{\Gamma(\gamma-\alpha)}(-z)^{-\alpha}{ }_{2} F_{1}\left(\alpha, \alpha-\gamma+1 ; \alpha-\beta+1 ; \frac{1}{z}\right) \\
& +\frac{\Gamma(\beta) \Gamma(\alpha-\beta)}{\Gamma(\gamma-\beta)}(-z)^{-\beta}{ }_{2} F_{1}\left(\beta, \beta-\gamma+1 ; \beta-\alpha+1 ; \frac{1}{z}\right) .(
\end{aligned}
$$

It holds when $|z|>1$ and $z$ is not a positive real number.

The other limit is $z \rightarrow 0$. In this paper we are interested in the arguments $a=1$, $b=3 / 2+2 k-i \mu$ and $c=3 / 2+k-i \mu$. Thus we have

$$
{ }_{2} F_{1}\left(1, \frac{3}{2}+2 k-i \mu ; \frac{3}{2}+k-i \mu ; z\right)=\sum_{n=0}^{\infty} \frac{\Gamma\left(\frac{3}{2}+2 k-i \mu+n\right) \Gamma\left(\frac{3}{2}+k-i \mu\right)}{\Gamma\left(\frac{3}{2}+2 k-i \mu\right) \Gamma\left(\frac{3}{2}+k-i \mu+n\right)} z^{n} .
$$

When we take the limit $\mu \rightarrow \infty, k \ll \mu$ and $n \ll \mu$, using (B3), we can resum the power series to get

$$
{ }_{2} F_{1}\left(1, \frac{3}{2}+2 k-i \mu ; \frac{3}{2}+k-i \mu ; z\right) \approx \sum_{n=0}^{\infty} z^{n}=\frac{1}{1-z} .
$$

The approximation holds because $|z|<1$ and the main contribution comes from the terms with small $n(\ll \mu)$, which makes it possible to push the summation upper bound to infinity. When we take $z \rightarrow 0$, we have

$$
{ }_{2} F_{1}(\alpha, \beta ; \gamma ; 0)=1
$$

which can also obtained by setting $z=0$ in the definition (B4).

\section{Appendix C: Hankel Function of Imaginary Order}

In this appendix we turn to the Hankel function of imaginary order $i \mu$, focusing on the large $\mu$ limit, which corresponds to the isocurvaton with a large mass. For an imaginary order, in contrast to the case discussed in Appendix A, we cannot neglect $J_{i \mu}$. We have

$$
\begin{aligned}
H_{i \mu}^{(1)}(x)= & \sum_{k=0}^{\infty} \frac{(-)^{k}}{k !} \frac{1+i \cot i \mu \pi}{\Gamma(k+1+i \mu)}\left(\frac{x}{2}\right)^{2 k+i \mu} \\
& -\frac{i}{\sin i \mu \pi} \sum_{k=0}^{\infty} \frac{(-)^{k}}{k !} \frac{1}{\Gamma(k-1+i \mu)}\left(\frac{x}{2}\right)^{2 k-i \mu} .
\end{aligned}
$$

We consider the large mass limit of (C1), that is, $\mu \rightarrow \infty$. First note that in this limit, we have

$$
\begin{aligned}
\cot i \mu \pi=-i \operatorname{coth} \mu \pi & \rightarrow-i, \\
\sin i \mu \pi=i \sinh \mu \pi & \rightarrow \frac{i e^{\mu \pi}}{2} .
\end{aligned}
$$


The above expressions imply that the second line of (C1) is exponentially suppressed. Besides, since the large $k$ terms in the summation of the first line of (C1) are also highly suppressed by the factor $(\Gamma(k+1) \Gamma(k+1-i \mu))^{-1}$, we expect the main contribution to this summation comes from the terms with small $k$. This permits us to apply $k \ll \mu$ in the Gamma function, and use the approximate form given in (B3),

$$
\Gamma(k+1+i \mu) \approx \sqrt{2 \pi} e^{i(\mu \ln \mu-\mu+k \pi / 4)} \mu^{k+1 / 2} e^{-\mu \pi / 2} .
$$

Therefore, we can pick out the $k$-dependence in the Gamma function, and resum the polynomial to get

$$
H_{i \mu}^{(1)} \approx e^{-i \mu(\ln \mu-1)} \sqrt{\frac{2 e^{\pi \mu}}{\pi \mu}} \exp \left[-\frac{x^{2}}{4 \mu} e^{-i \frac{\pi}{4}}\right]\left(\frac{x}{2}\right)^{i \mu} .
$$

[1] A. H. Guth, "The Inflationary Universe: A Possible Solution To The Horizon And Flatness Problems," Phys. Rev. D 23, 347 (1981);

K. Sato, "First Order Phase Transition Of A Vacuum And Expansion Of The Universe," Mon. Not. Roy. Astron. Soc. 195, 467 (1981).

[2] A. A. Starobinsky, "A new type of isotropic cosmological models without singularity," Phys. Lett. B 91, 99 (1980);

A. D. Linde, "A New Inflationary Universe Scenario: A Possible Solution Of The Horizon, Flatness, Homogeneity, Isotropy And Primordial Monopole Problems," Phys. Lett. B 108, 389 (1982);

A. Albrecht and P. J. Steinhardt, "Cosmology For Grand Unified Theories With Radiatively Induced Symmetry Breaking," Phys. Rev. Lett. 48, 1220 (1982).

[3] E. Komatsu et al., "Seven-Year Wilkinson Microwave Anisotropy Probe (WMAP) Observations: Cosmological Interpretation," arXiv:1001.4538 [astro-ph.CO].

[4] L. Susskind, "The Anthropic landscape of string theory," In *Carr, Bernard (ed.): Universe or multiverse?* 247-266 hep-th/0302219].

[5] J. S. Schwinger, "The Special Canonical Group", Proc. Nat. Acad. Sci. 46, 1401 (1960); P. M. Bakshi and K. T. Mahanthappa, "Expectation value formalism in quantum field theory. 1," J. Math. Phys. 4, 1 (1963); P. M. Bakshi and K. T. Mahanthappa, "Expectation value formalism in quantum field theory. 2," J. Math. Phys. 4, 12 (1963); L. V. Keldysh, Zh. Eksp. Teor. Fiz. 47, 1515 (1964).

[6] S. Weinberg, "Quantum contributions to cosmological correlations," Phys. Rev. D 72, 043514 (2005). arXiv:hep-th/0506236].

[7] J. M. Maldacena, "Non-Gaussian features of primordial fluctuations in single field inflationary models," JHEP 0305, 013 (2003) arXiv:astro-ph/0210603.

[8] T. S. Bunch and P. C. W. Davies, "Quantum Field Theory In De Sitter Space: Renormalization By Point Splitting," Proc. Roy. Soc. Lond. A 360, 117 (1978).

[9] A. R. Liddle, A. Mazumdar and F. E. Schunck,Dimopoulos:2005ac "Assisted inflation," Phys. Rev. D 58, 061301 (1998) arXiv:astro-ph/9804177.

[10] E. Silverstein and D. Tong, "Scalar speed limits and cosmology: Acceleration from Dcceleration," Phys. Rev. D 70, 103505 (2004) arXiv:hep-th/0310221;

[11] M. X. Huang, G. Shiu and B. Underwood, "Multifield DBI Inflation and Non-Gaussianities," Phys. Rev. D 77, 023511 (2008) arXiv:0709.3299 [hep-th]]. 
[12] J. Ward, "DBI N-flation," JHEP 0712, 045 (2007) [arXiv:0711.0760 [hep-th]].

[13] S. Pi and D. Wang, "Cosmological perturbations in inflation with multiple sound speeds," Nucl. Phys. B 862, 409 (2012) [arXiv:1107.0813 [hep-th]].

[14] M. Sasaki and T. Tanaka, "Superhorizon scale dynamics of multiscalar inflation," Prog. Theor. Phys. 99, 763 (1998) [gr-qc/9801017].

[15] C. Gordon, D. Wands, B. A. Bassett and R. Maartens, "Adiabatic and entropy perturbations from inflation," Phys. Rev. D 63, 023506 (2001) arXiv:astro-ph/0009131.

[16] L. Amendola, C. Gordon, D. Wands and M. Sasaki, "Correlated perturbations from inflation and the cosmic microwave background," Phys. Rev. Lett. 88, 211302 (2002) arXiv:astro-ph/0107089.

[17] C. M. Peterson and M. Tegmark, "Testing Two-Field Inflation," Phys. Rev. D 83, 023522 (2011) arXiv:1005.4056 [astro-ph.CO]].

[18] C. Armendariz-Picon, T. Damour and V. F. Mukhanov, "k-Inflation," Phys. Lett. B 458, 209 (1999) arXiv:hep-th/9904075.

[19] X. Gao, "On Cross-correlations between Curvature and Isocurvature Perturbations during Inflation," JCAP 1002, 019 (2010) arXiv:0908.4035 [hep-th]].

[20] V. F. Mukhanov and G. V. Chibisov, "The Vacuum energy and large scale structure of the universe," Sov. Phys. JETP 56, 258 (1982) [Zh. Eksp. Teor. Fiz. 83, 475 (1982)].

[21] M. Sasaki, "Large Scale Quantum Fluctuations in the Inflationary Universe," Prog. Theor. Phys. 76, 1036 (1986).

[22] V. F. Mukhanov, "Quantum Theory of Gauge Invariant Cosmological Perturbations," Sov. Phys. JETP 67, 1297 (1988) [Zh. Eksp. Teor. Fiz. 94N7, 1 (1988)].

[23] M. Sasaki and E. D. Stewart, "A General Analytic Formula For The Spectral Index Of The Density Perturbations Produced During Inflation," Prog. Theor. Phys. 95, 71 (1996) arXiv:astro-ph/9507001.

[24] D. H. Lyth, K. A. Malik and M. Sasaki, "A general proof of the conservation of the curvature perturbation," JCAP 0505, 004 (2005) arXiv:astro-ph/0411220.

[25] A. A. Starobinsky, "Multicomponent de Sitter (Inflationary) Stages and the Generation of Perturbations," JETP Lett. 42, 152 (1985) [Pisma Zh. Eksp. Teor. Fiz. 42, 124 (1985)].

[26] A. A. Starobinsky, "Dynamics Of Phase Transition In The New Inflationary Universe Scenario And Generation Of Perturbations," Phys. Lett. B 117, 175 (1982).

[27] A. D. Linde, "Hybrid inflation," Phys. Rev. D 49, 748 (1994) arXiv:astro-ph/9307002.

[28] D. H. Lyth, "Issues concerning the waterfall of hybrid inflation," arXiv:1005.2461 [astroph.CO].

[29] D. H. Lyth, "The contribution of the hybrid inflation waterfall to the primordial curvature perturbation," arXiv:1012.4617 [astro-ph.CO].

[30] J. Fonseca, M. Sasaki and D. Wands, "Large-scale Perturbations from the Waterfall Field in Hybrid Inflation," JCAP 1009, 012 (2010) [arXiv:1005.4053 [astro-ph.CO]].

[31] A. A. Abolhasani, H. Firouzjahi and M. H. Namjoo, "Curvature Perturbations and nonGaussianities from Waterfall Phase Transition during Inflation," Class. Quant. Grav. 28, 075009 (2011) [arXiv:1010.6292 [astro-ph.CO]].

[32] J. O. Gong and M. Sasaki, "Waterfall field in hybrid inflation and curvature perturbation," JCAP 1103, 028 (2011) [arXiv:1010.3405 [astro-ph.CO]].

[33] A. A. Abolhasani, H. Firouzjahi, S. Khosravi and M. Sasaki, "Local Features with Large Spiky non-Gaussianities during Inflation," arXiv:1204.3722 [astro-ph.CO].

[34] X. Chen and Y. Wang, "Quasi-Single Field Inflation and Non-Gaussianities," JCAP 1004, 
027 (2010) arXiv:0911.3380 [hep-th]].

[35] X. Chen and Y. Wang, "Large non-Gaussianities with Intermediate Shapes from Quasi-Single Field Inflation," Phys. Rev. D 81, 063511 (2010) [arXiv:0909.0496 [astro-ph.CO]].

[36] A. J. Tolley and M. Wyman, "The Gelaton Scenario: Equilateral non-Gaussianity from multifield dynamics," Phys. Rev. D 81, 043502 (2010) arXiv:0910.1853 [hep-th]].

[37] S. Cremonini, Z. Lalak and K. Turzynski, "Strongly Coupled Perturbations in Two-Field Inflationary Models," JCAP 1103, 016 (2011) arXiv:1010.3021 [hep-th]].

[38] A. Achucarro, J. O. Gong, S. Hardeman, G. A. Palma and S. P. Patil, "Features of heavy physics in the CMB power spectrum," JCAP 1101, 030 (2011) [arXiv:1010.3693 [hep-ph]].

[39] G. Shiu and J. Xu, "Effective Field Theory and Decoupling in Multi-field Inflation: An Illustrative Case Study," Phys. Rev. D 84, 103509 (2011) arXiv:1108.0981 [hep-th]].

[40] S. Cespedes, V. Atal and G. A. Palma, "On the importance of heavy fields during inflation," arXiv:1201.4848 [hep-th].

[41] A. Achucarro, J. O. Gong, S. Hardeman, G. A. Palma and S. P. Patil, "Effective theories of single field inflation when heavy fields matter," arXiv:1201.6342 [hep-th].

[42] A. Avgoustidis, S. Cremonini, A. -C. Davis, R. H. Ribeiro, K. Turzynski and S. Watson, "Decoupling Survives Inflation: A Critical Look at Effective Field Theory Violations During Inflation," arXiv:1203.0016 [hep-th].

[43] V. Assassi, D. Baumann and D. Green, "On Soft Limits of Inflationary Correlation Functions," arXiv:1204.4207 [hep-th].

[44] X. Chen and Y. Wang, JCAP 1209, 021 (2012) arXiv:1205.0160 [hep-th]].

[45] X. Gao, D. Langlois and S. Mizuno, arXiv:1205.5275 [hep-th]. 